\title{
Multiple interactions and generalized parton distributions
}

\section{Markus Diehl*}

Deutsches Elektronen-Synchroton DESY, 22603 Hamburg, Germany

E-mail: markus.diehledesy.de

\begin{abstract}
Multiple parton interactions in a single proton-proton collision are expected to play an important role for many observables at LHC. To a large part their phenomenological description relies on rather simple and physically intuitive assumptions. We report on an investigation of multiple hard interactions in QCD, which aims at identifying to which extent this simple description arises from theory and where it needs to be extended. An approximate connection with generalized parton distributions may help elucidate some aspects of multiple-interaction dynamics.
\end{abstract}

XVIII International Workshop on Deep-Inelastic Scattering and Related Subjects, DIS 2010 April 19-23, 2010

Firenze, Italy

${ }^{*}$ Speaker. 


\section{Introduction}

In proton-proton collisions at very high energies one can have events in which several partons in one proton scatter on partons in the other proton and produce particles of large transverse momentum or large mass. The effects of such multiparton interactions average out in sufficiently inclusive observables, which can be described by conventional factorization formulae that involve a single hard scattering. Multiple interactions do however change the structure of the final state and may be important for many analyses at LHC [1]. A brief review on the subject can be found in [2].

Phenomenological estimates of multiparton dynamics rely on models that are physically intuitive but rather simplified, whereas a systematic description in QCD remains a challenge. The present contribution reports on some steps towards this goal. The discussion is limited to tree-level graphs, which already exhibit important features. Loop corrections, in particular soft gluon exchange, add a further layer of complexity and are essential for determining if and in which form factorization holds. For details and further discussion we refer to [3].

\section{Cross section formula and multiparton distributions}

An example process where multiparton interactions contribute is the production of two electroweak gauge bosons ( $W, Z$ or $\gamma^{*}$ with large virtuality) in kinematics where the transverse momenta of the bosons are small compared with their mass or virtuality. Since we are interested in the details of the final state, we keep the cross section differential in the transverse boson momenta. For the corresponding process with a single boson, a powerful theory in terms of transverse-momentum dependent parton densities has been developed [ 4 , which one may hope to generalize to the case of multiple hard scattering.

Figure 11a shows a graph with two hard-scattering subprocesses. The transverse momentum of each produced boson is the sum of the transverse momenta of a quark and an antiquark. With two partons emitted by each proton the transverse parton momenta are in general not equal in the scattering amplitude and its conjugate, as illustrated in the figure. Momentum conservation implies $\mathbf{r}+\overline{\mathbf{r}}=\mathbf{0}$ for the differences between the parton momenta in the amplitude and its conjugate. Performing a Fourier transform w.r.t. $\mathbf{r}$ and $\overline{\mathbf{r}}$ one finds that their Fourier conjugate variables are equal, $\mathbf{y}=\overline{\mathbf{y}}$.

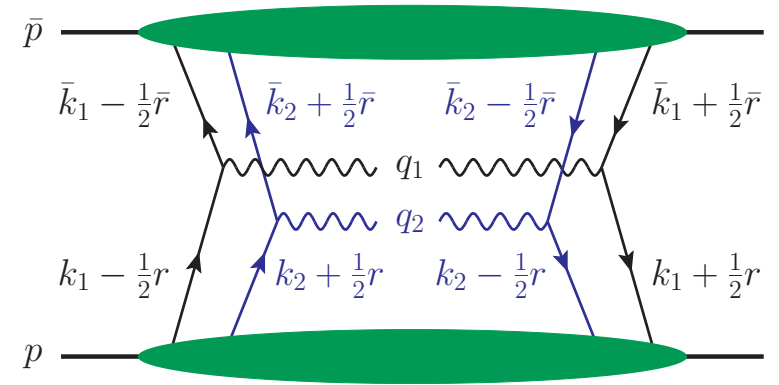

(a)

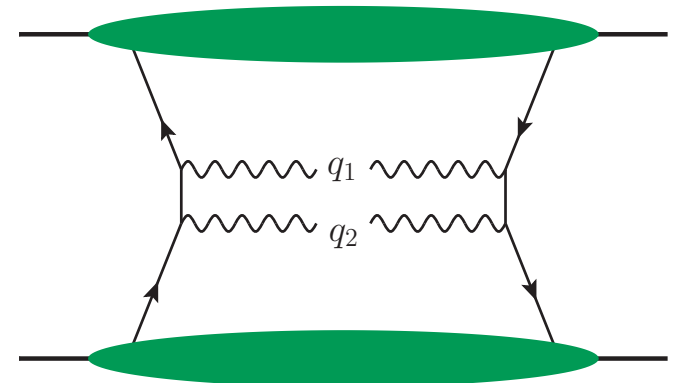

(b)

Figure 1: Graphs for the production of two gauge bosons by double (a) or single (b) hard scattering. 
Making the usual kinematic approximations in the hard-scattering subprocesses (i.e. neglecting small momentum components compared with large ones) one obtains

$$
\begin{aligned}
& \left.\frac{d \sigma}{\prod_{i=1}^{2} d x_{i} d \bar{x}_{i} d^{2} \mathbf{q}_{i}}\right|_{\text {Fig. 四 } a}=\frac{1}{S} \sum_{\substack{a_{1}, a_{2}=q, \Delta q, \delta q \\
\bar{a}_{1}, \bar{a}_{2}=\bar{q}, \Delta \bar{q}, \delta \bar{q}}} \hat{\sigma}_{a_{1} \bar{a}_{1}}\left(\hat{s}=x_{1} \bar{x}_{1} s\right) \hat{\sigma}_{a_{2} \bar{a}_{2}}\left(\hat{s}=x_{2} \bar{x}_{2} s\right) \\
& \times\left[\prod_{i=1}^{2} \int d^{2} \mathbf{k}_{i} d^{2} \overline{\mathbf{k}}_{i} \delta^{(2)}\left(\mathbf{q}_{i}-\mathbf{k}_{i}-\overline{\mathbf{k}}_{i}\right)\right] \int d^{2} \mathbf{y} F_{a_{1}, a_{2}}\left(x_{i}, \mathbf{k}_{i}, \mathbf{y}\right) F_{\bar{a}_{1}, \bar{a}_{2}}\left(\bar{x}_{i}, \overline{\mathbf{k}}_{i}, \mathbf{y}\right)
\end{aligned}
$$

with $S=2$ if the produced bosons are identical and $S=1$ if they are not. $\hat{\sigma}_{a_{i} \bar{a}_{i}}$ denotes the hardscattering cross section for single-boson production, and the kinematic variables are $s=(p+\bar{p})^{2}$, $x_{i}=\left(q_{i} \bar{p}\right) /(p \bar{p})$ and $\bar{x}_{i}=\left(q_{i} p\right) /(\bar{p} p)$. The definition of double-parton distributions closely resembles the one for the transverse-momentum dependent distribution of a single quark [ [ two-quark distribution in the proton with momentum $p$ we have

$$
\begin{aligned}
F_{a_{1}, a_{2}}\left(x_{i}, \mathbf{k}_{i}, \mathbf{y}\right)= & {\left[\prod_{i=1}^{2} \int \frac{d z_{i}^{-} d^{2} \mathbf{z}_{i}}{(2 \pi)^{3}} e^{i x_{i} z_{i}^{-} p^{+}-i \mathbf{z}_{i} \mathbf{k}_{i}}\right] 2 p^{+} \int d y^{-} } \\
& \times\left.\left\langle p\left|\bar{q}\left(-\frac{1}{2} z_{2}\right) \Gamma_{a_{2}} q\left(\frac{1}{2} z_{2}\right) \bar{q}\left(y-\frac{1}{2} z_{1}\right) \Gamma_{a_{1}} q\left(y+\frac{1}{2} z_{1}\right)\right| p\right\rangle\right|_{z_{1}^{+}=z_{2}^{+}=y^{+}=0},
\end{aligned}
$$

where we use light-cone coordinates $u^{ \pm}=\left(u^{0} \pm u^{3}\right) / \sqrt{2}$ for each four-vector $u$. One may regard $\mathbf{k}_{i}$ as the "average" transverse momentum of each quark and $\mathbf{y}$ as their "average" transverse distance from each other, where the "average" refers to the scattering amplitude and its conjugate. Regarding the transverse coordinates $F\left(x_{i}, \mathbf{k}_{i}, \mathbf{y}\right)$ has the structure of a Wigner distribution [5], which depends on both momentum and position variables for each particle. (This does not contradict the uncertainty principle since Wigner distributions are not probability densities.) A similar application of this concept has been discussed for generalized parton distributions in [6].

Integrating (2.1) over the transverse boson momenta $\mathbf{q}_{1}$ and $\mathbf{q}_{2}$ one obtains

$$
\begin{aligned}
\left.\frac{d \sigma}{\prod_{i=1}^{2} d x_{i} d \bar{x}_{i}}\right|_{\text {Fig. 目a } a}= & \frac{1}{S} \sum_{\substack{a_{1}, a_{2}=q, \Delta q, \delta q \\
\bar{a}_{1}, \bar{a}_{2}=\bar{q}, \Delta \bar{q}, \delta \bar{q}}} \hat{\sigma}_{a_{1} \bar{a}_{1}}\left(\hat{s}=x_{1} \bar{x}_{1} s\right) \hat{\sigma}_{a_{2} \bar{a}_{2}}\left(\hat{s}=x_{2} \bar{x}_{2} s\right) \\
& \times \int d^{2} \mathbf{y} F_{a_{1}, a_{2}}\left(x_{i}, \mathbf{y}\right) F_{\bar{a}_{1}, \bar{a}_{2}}\left(\bar{x}_{i}, \mathbf{y}\right) .
\end{aligned}
$$

The transverse-momentum integrated distribution $F_{a_{1}, a_{2}}\left(x_{i}, \mathbf{y}\right)=\int d^{2} \mathbf{k}_{1} d^{2} \mathbf{k}_{2} F_{a_{1}, a_{2}}\left(x_{i}, \mathbf{k}_{i}, \mathbf{y}\right)$ may be interpreted as the probability density for finding two quarks with momentum fractions $x_{1}$ and $x_{2}$ at a relative transverse distance $\mathbf{y}$ in the proton. The form (2.3) has long been known (see e.g. [7], 8]) and underlies most phenomenological models for multiparton interactions.

For each quark there are three relevant Dirac matrices in (2.2),

$$
\Gamma_{q}=\frac{1}{2} \gamma^{+}, \quad \Gamma_{\Delta q}=\frac{1}{2} \gamma^{+} \gamma_{5}, \quad \Gamma_{\delta q}^{j}=\frac{1}{2} i \sigma^{j+} \gamma_{5} \text { with } j=1,2,
$$

which respectively project on unpolarized, longitudinally polarized and transversely polarized quarks. Note that polarized two-parton distributions appear even in an unpolarized proton, since they describe spin correlations between the two partons. For small but comparable $x_{1}$ and $x_{2}$ one 
may well have sizeable spin correlations between the two quarks (which are close in rapidity for $x_{1} \sim x_{2}$ ), even if there is little correlation between the polarizations of a quark and the proton (which are far apart in rapidity). The relevance of such correlations in multiparton interactions was pointed out already in [8] but has to our knowledge never been included in phenomenological estimates.

Note that if parton spin correlations are sizeable they can have a strong impact on observables. For the production of two gauge bosons one can easily see that the product $F_{\Delta q, \Delta q} F_{\Delta \bar{q}, \Delta \bar{q}}$ of longitudinal spin correlations enters the cross section with the same weight as the unpolarized term $F_{q, q} F_{\bar{q}, \bar{q}}$. One also finds that product $F_{\delta q, \delta q} F_{\delta \bar{q}, \delta \bar{q}}$ of transverse spin correlations give rise to a $\cos (2 \varphi)$ modulation in the angle $\varphi$ between the decay planes of the two bosons and thus affects the distribution of final-state particles.

The formulae given so far have ignored the color structure of the multiparton distributions. The quark lines with momenta $k_{1} \pm \frac{1}{2} r$ in Fig. 11a can couple to a color singlet (as in single-parton distributions) but they can also couple to a color octet, provided that the lines with momenta $k_{2} \pm \frac{1}{2} r$ are coupled to a color-octet as well. Such color-octet distributions contribute to the multiple-scattering cross section, as was already pointed out in [8]. A more detailed discussion will be given in [3].

\section{Power behavior}

It is easy to determine the power behavior of the cross section formula (2.1) for two hard scatters. The hard-scattering cross sections $\hat{\sigma}$ behave like $1 / Q^{2}$, where $Q^{2} \sim q_{1}^{2} \sim q_{2}^{2}$ denotes the size of the large squared mass or virtuality of the gauge bosons. With transverse momenta $\mathbf{q}_{1} \sim \mathbf{q}_{2}$ of generic hadronic size $\Lambda$ we find

$$
\left.\frac{d \sigma}{\prod_{i=1}^{2} d x_{i} d \bar{x}_{i} d^{2} \mathbf{q}_{i}}\right|_{\text {Fig. 目 } a} \sim \frac{1}{Q^{4} \Lambda^{2}}
$$

where we have used that the two-parton distributions scale like $F \sim 1 / \Lambda^{2}$ and that the typical transverse distance $\mathbf{y}$ between the partons is of order $1 / \Lambda$. The same power behavior as in (3.1) is obtained for the case where both bosons are produced in a single hard scattering, as shown in Fig. [ ] b. For the cross section differential in the transverse boson momenta, multiple hard interactions are therefore not power suppressed.

The situation changes when one integrates over $\mathbf{q}_{1}$ and $\mathbf{q}_{2}$. In the double-scattering mechanism both transverse momenta are restricted to be of size $\Lambda$, but for a single hard scattering one has $\left|\mathbf{q}_{1}+\mathbf{q}_{2}\right| \sim \Lambda$ whereas the individual transverse momenta can be as large as $Q$. Because of this phase space effect one has

$$
\left.\frac{d \sigma}{\prod_{i=1}^{2} d x_{i} d \bar{x}_{i}}\right|_{\text {Fig. 目 } a} \sim \frac{\Lambda^{2}}{Q^{4}},\left.\quad \quad \frac{d \sigma}{\prod_{i=1}^{2} d x_{i} d \bar{x}_{i}}\right|_{\text {Fig. 目 } p} \sim \frac{1}{Q^{2}} .
$$

In the transverse-momentum integrated cross section, multiple hard scattering is therefore only a power correction. This is required for the validity of the usual factorization formulae, which describe only the single-scattering contribution. 


\section{Connection with generalized parton distributions}

A simple ansatz for modeling two-parton distributions is to write them as a product of singleparton distributions, thus neglecting correlations between the two partons. This provides a starting point for phenomenology, even though one may not expect such an approximation to be very precise. A way to implement this ansatz for the distributions $F\left(x_{i}, \mathbf{k}_{i}, \mathbf{y}\right)$ is to insert a sum $\sum_{X}|X\rangle\langle X|$ over all physical states between the two bilinear operators in (2.2). If one assumes that the proton state is dominant in this sum, one has

$$
\left\langle p\left|\left(\bar{q}_{x_{2}} q_{x_{2}}\right)\left(\bar{q}_{x_{1}} q_{x_{1}}\right)\right| p\right\rangle \approx \int \frac{d p^{++}}{2 p^{\prime+}} \frac{d^{2} \mathbf{p}^{\prime}}{(2 \pi)^{3}}\left\langle p\left|\bar{q}_{x_{2}} q_{x_{2}}\right| p^{\prime}\right\rangle\left\langle p^{\prime}\left|\bar{q}_{x_{1}} q_{x_{1}}\right| p\right\rangle,
$$

where the subscripts $x_{1}, x_{2}$ indicate the momentum fraction associated with each field and spinor indices have been omitted for brevity. After the Fourier transform in (2.2), momentum conservation imposes $p^{\prime+}=p^{+}$but one still has $\mathbf{p}^{\prime} \neq \mathbf{p}$. The two-parton distribution $F\left(x_{i}, \mathbf{k}_{i}, \mathbf{y}\right)$ is thus approximated by a product of two generalized parton distributions with zero skewness $\xi$. For transverse-momentum integrated distributions one obtains a simple representation $F\left(x_{i}, \mathbf{y}\right)=$ $\int d^{2} \mathbf{b} f\left(x_{2}, \mathbf{b}\right) f\left(x_{1}, \mathbf{b}+\mathbf{y}\right)$ in terms of impact parameter dependent parton densities [9].

The same method can be applied to the color-octet distributions mentioned above. Rearranging the quark operators as

$$
\frac{1}{2}\left(\bar{q}_{x_{2}} \lambda^{a} q_{x_{2}}\right)\left(\bar{q}_{x_{1}} \lambda^{a} q_{x_{1}}\right)=-\left(\bar{q}_{x_{1}} q_{x_{2}}\right)\left(\bar{q}_{x_{2}} q_{x_{1}}\right)-\frac{1}{3}\left(\bar{q}_{x_{2}} q_{x_{2}}\right)\left(\bar{q}_{x_{1}} q_{x_{1}}\right),
$$

where $\lambda^{a}$ are the Gell-Mann matrices, one can insert proton states between the operators in parentheses on the r.h.s. For the first term this leads to generalized parton distributions with nonzero skewness $\xi$, since the fields coupled to a color singlet carry different longitudinal momenta.

Although it is only approximate, the connection with generalized parton distributions that can be measured in exclusive reactions - pointed out already in [10] - will hopefully help to better understand at least some features of multiple hard interactions.

\section{References}

[1] H. Jung et al., arXiv:0903.3861 [hep-ph];

S. Alekhin et al., hep-ph/0601012 and hep-ph/0601013.

[2] T. Sjöstrand, P. Z. Skands, JHEP 0403 (2004) 053 [hep-ph/0402078].

[3] M. Diehl, D. Ostermeier, A. Schäfer, in preparation.

[4] J. C. Collins, D. E. Soper, Nucl. Phys. B193 (1981) 381;

X. D. Ji, J. P. Ma, F. Yuan, Phys. Lett. B597 (2004) 299 [hep-ph/0405085];

J. C. Collins, T. C. Rogers, A. M. Stasto, Phys. Rev. D77 (2008) 085009 [arXiv:0708.2833 [hep-ph]].

[5] M. Hillery, R. F. O’Connell, M. O. Scully, E. P. Wigner, Phys. Rept. 106 (1984) 121.

[6] A. V. Belitsky, X. D. Ji, F. Yuan, Phys. Rev. D69 (2004) 074014 [hep-ph/0307383].

[7] N. Paver, D. Treleani, Z. Phys. C28 (1985) 187.

[8] M. Mekhfi, Phys. Rev. D32 (1985) 2371; Phys. Rev. D 32 (1985) 2380.

[9] M. Burkardt, Int. J. Mod. Phys. A18 (2003) 173 [hep-ph/0207047].

[10] L. Frankfurt, M. Strikman, C. Weiss, Phys. Rev. D69 (2004) 114010 [hep-ph/0311231]. 